\title{
INFECTIVIDAD Y DIVERSIDAD DE HONGOS MICORRICICOS ARBUSCULARES DE LA RIZOSFERA DE LOS TALARES DE MAGDALENA, PROVINCIA DE BUENOS AIRES, ARGENTINA
}

\author{
(Infectivity and diversity of mycorrhizal arbuscular fungi in rhizosphere of \\ Magdalena talares, Buenos Aires Province, Argentina)
}

\author{
Gabriela Irrazabal $^{1,3}$, Silavana Velázquez $^{2,3}$ \& Marta Cabello $^{2,3}$ \\ 'Universidad Nacional de La Plata \\ ${ }^{2}$ Comisión de Investigaciones Científicas de la provincia de Buenos Aires \\ ${ }^{3}$ Instituto Spegazzini, Av. 53 No 477, B1900AVJ, La Plata \\ E-mail: mcabello@netverk.com.ar
}

Palabras clave: micorrizas arbusculares, infectividad, diversidad, rendol, talares, Celtis tala,Scutia buxifolia Key words: arbuscular mycorrhizal fungi, infectivity, diversity, rendol, talares, Celtis tala, Scutia buxifolia

\section{RESUMEN}

Los hongos micorrícicos arbusculares son considerados microorganismos claves, particularmente en ambientes donde las condiciones edafo-climáticas son severas. Los talares, bosques xerícos dominados por Celtis tala(tala) y Scutia buxifolia (coronillo), comprenden la comunidad boscosa más importante de la región pampeana. Se desarrollan sobre cordones conchiles depositados durante las ingresiones marinas del Cuaternario, en suelos Rendoles.

Se estimó la infectividad y diversidad de hongos micorrícicos de 5 situaciones: bosque puro de tala, bosque puro de coronillo, bosque con codominancia de ambas especies y dos áreas desmontadas.

La infectividad se valoró mediante bioensayos en invernáculo. Se emplearon diluciones de suelo (1:0; 1:4 y 1:40) y alfalfa (Medicago sativa) como planta hospedadora. Los resultados se relacionaron con el número de esporas presentes en la rizósfera. La biodiversidad se estimó mediante la identificación de las especies de hongos micorrizicos-arbusculares presentes, cuantificando la densidad especifica de sus esporas. Se calculó el índice de biodiversidad de Shannon-Weaver.

La colonización presentó valores altos y estables en todas las diluciones y situaciones; el número de esporas fue significativamente mayor en los desmontes. Se identificaron 26 especies de hongos micorrícicos arbusculares pertenecientes en su mayoría a Glomeraceae $y$ Acaulosporaceae, pocas especies pertenecieron a Gigasporaceae. Glomus clarum, Acaulospora delicata, Acaulospora sp. 1, A. mellea, Glomus constrictum y G. coronatum, fueron las especies con mayor abundancia relativa. La biodiversidad fue mayor en los bosques con codominancia de tala y coronillo y puro de coronillo; los desmontes presentaron un valor más bajo y el bosque puro de tala presentó la menor diversidad fúngica del área.

La diferentes situaciones afectaron directamente la densidad relativa de las especies fúngicas, la distribución de las familias y la biodiversidad.

\section{ABSTRACT}

Arbuscular mycorrhizal fungi are considered as key organisms mainly in environments where edapho-climatic conditions are severe.Talares, xeric forests dominated by Celtis tala (tala) and Scutia buxifolia (coronillo) form the most important woody area in the eastern plain called Pampa in Argentina. They grow on shelly ridges deposited after the sea ingressions of the Quaternary on rendol soils.

The infectivity and diversity of mycorrhizal fungi in 5 situations were estimated: Celtis tala pure forest, Scutia buxifolia pure forest, forest with codominance of both species and two cleared areas. Infectivity was measured by means of bioassays in greenhouse. Soil dilutions (1: 0;1:4 and 1:40) and alfalfa (Medicago sativa) as a host plant were used. Results were related to the number of spores present in the rhizosphere. Biodiversity was estimated according 
to the identification of the species of arbuscular mycorrhizal fungi present by quantifying the specific density of their spores. Shanon-Weaver biodiversity index was calculated.

Colonies exhibited high and stable values in every dilution and situation; number of spores was significantly higher in cleared areas. Twenty six species of arbuscular mycorrhizal fungi belonging most of them to Glomeraceae and Acaulosporaceae were identified. A few species belonged to Gigasporaceae. Glomus clarum, Acaulospora delicata, Acaulospora sp.1, A. mellea, Glomus constrictum and G.coronatum were species with the highest relative abundance.

Biodiversity was higher in forests with codominance of Celtis tala and Scutia buxifolia in pure forest of Scutia buxifolia; cleared areas showed lower values while pure forest of Celtis tala exhibited the least fungal diversity of the area. Different situations affected directly the relative density of fungal species, the distribution of families and biodiversity.

\section{INTRODUCCION}

Los hongos formadores de micorrizas arbusculares están presentes en los ecosistemas más diversos. Las asociaciones micorrícicas son factores potenciales en la determinación de la diversidad en los sistemas naturales: éstas, probablemente, pueden modificar la estructura y el funcionamiento de las comunidades de plantas de una manera compleja e impredecible (Grime et al., 1987). Cualquier cambio en la población de hongos formadores de micorrizas producirá efectos sobre la composición en la comunidad de plantas (supervivencia, competencia, distribución florística), causando cambios en la biología de los ecosistemas (Miller \& Allen, 1992; Molina et al., 1992). Por otro lado, la composición de la comunidad de plantas puede afectar a la comunidad fúngica, causando variaciones en las tasas de reproducción y supervivencia.

Los hongos micorrícicos-arbusculares forman una simbiosis muy difundida y son considerados microorganismos claves, particularmente en ambientes severos donde las condiciones edafo-climáticas son críticas y estresantes para la mayoría de los microorganismos (Allen, 1991). Dentro de un ecosistema cumplen múltiples funciones; son importantes en las absorción de fósforo en la mayoría de las plantas terrestres, especialmente en suelos con poca disponibilidad de este elemento (Hetrick, 1984), permiten la absorción de micronutrientes relativamente inmóviles en la solución del suelo ( $\mathrm{Zn}$ y $\mathrm{Cu}$ ), promueven el desarrollo de la planta al mejorar la resistencia a factores de estrés bióticos y abióticos.
Además, influencian positivamente las tasas de movimiento del agua hacia el interior y el exterior de la planta hospedadora, con consecuentes efectos en la hidratación de los tejidos y la fisiología de las hojas, la conductancia estomática y la transpiración, aumentando visiblemente las tasas fotosintéticas (Augé, 2001).

La ocurrencia y diversidad de los hongos micorrícicos está relacionada con sus requerimientos edáficos, los cuales pueden afectar la germinación de las esporas, la colonización y la efectividad (Sieverding, 1991). Uno de los factores decisivos es el pH, aunque también existen variaciones relacionadas con la textura, salinidad, temperatura, concentración de nutrientes y presencia de metales pesados, entre otros. Existen numerosos trabajos que señalan la disminución de la diversidad de estos hongos a medida que las actividades humanas se incrementan (Johnson \& Pfleger, 1992).

La infectividad de un suelo, se define como la habilidad de los propágulos de hongos arbusculares para iniciar la colonización micorrícica en plantas hospedadoras (Bouhot, 1980). La infectividad está relacionada con el número de propágulos presentes en el suelo en forma de esporas, micelio externo y segmentos colonizados de raíces.

Los talares son bosques xéricos dominados por Celtis tala Gil ex Planch. ("tala") y Scutia buxifolia Reiss ("coronillo"); comprenden la comunidad boscosa más importante de la región pampeana. En el Partido de Magdalena (Prov. de Buenos Aires, Argentina) se encuentra la parte mejor conservada del bosque nativo. La heterogeneidad ambiental del área determina la variación en la composición de la vegetación (Cabrera, 1968). Los cordones boscosos se ubican paralelos a la costa del Río de La Plata. El suelo está formado a partir de material calcáreo de origen biológico, valvas de moluscos depositadas durante ingresiones marinas del Cuaternario (Fidalgo et al., 1975). En sentido transversal a la costa, se observa una variación espacial claramente definida, en la cual los cordones más alejados al río presentan dominancia de $C$. tala, en los cordones cercanos domina $S$. buxifolia y en los intermedios existe una codominancia de ambos (Goya et al., 1992). Esta distribución, determina una variación en la composición herbácea, no sólo entre los distintos cordones, sino posiblemente dentro de un mismo cordón, según la ubicación en el interior del bosque, ecotono y pastizal graminoso.

El suelo de los cordones ha sido clasificado como Rendol típico, mientras que en los intercordones se desarrolla la pradera salada, con suelos aluviales de texturas contrastantes con un alto contenido de sodio y la pradera húmeda con drenaje imperfecto y suelos hidromórficos no alcalinos (Sanchez et al., 1976).

El objetivo de este estudio fue estimar la infec- 
tividad y la diversidad de hongos formadores de micorrizas arbusculares de suelos correspondientes a: bosque puro de tala (Celtis tala Gill. ex Planch.), bosque puro de coronillo (Scutia buxifolia Reiss.), bosque con codominancia de ambas especies y dos áreas desmontadas.

\section{MATERIALES Y METODOS}

Area de muestreo. La zona de estudio se encuentra a 20 $\mathrm{km}$ hacia el SE de la localidad de Magdalena, provincia de Buenos Aires. El clima regional presenta una temperatura media anual de $15,9^{\circ} \mathrm{C}$ y precipitaciones de $885 \mathrm{~mm}$ por año (Servicio Meteorológico Nacional, 1986).

Diseño experimental. Las situaciones muestreadas fueron: bosque dominado por tala, bosque dominado por coronillo, bosque con codominancia de ambas especies y 2 áreas desmontadas (desmonte antiguo más de 15 años y desmonte reciente menos de 4 años). Cada situación tuvo tres réplicas ubicadas en distintos cordones, siguiendo un diseño en bloques completos. Un bloque se situó en el sector de cordones más alejado de la costa a aproximadamente $3000 \mathrm{~m}$ de la costa del Río de La Plata. Los dos bloques restantes se ubicaron en cordones localizados en posiciones intermedias entre los $3000 \mathrm{y}$ $2000 \mathrm{~m}$ desde la costa del río. De cada situación se tomaron 6 submuestras las cuales fueron homogeneizadas en una sola muestra; cada una de estas muestras completas corresponde a un albardón con bosques puros, codominantes y desmontes. Las muestras se tomaron con pala del suelo de la rizósfera correspondiente a la vegetación tanto herbácea como arbórea, hasta $15 \mathrm{~cm}$ de profundidad.

Bioensayo de infectividad. Para determinar la infectividad de los suelos de las distintas situaciones analizadas se efectuó un muestreo de la rizósfera (febrero 2004) el cual fue utilizado para estimar los propágulos viables presentes en el suelo como esporas, micelio extraradical y segmentos de raíces colonizados con micelio intraradical y vesículas. Se procedió a establecer el número de esporas presentes en 100gr de suelo seco utilizando el método de tamizado húmedo - decantación (Gerdemann \& Nicolson, 1963) y centrifugación en gradiente de sacarosa (Walker et al.,1982). Esta estimación se hizo para relacionar el número de esporas presentes en la muestra con la colonización de las raíces. Las restantes formas de propágulos (micelio extraradical y segmentos de raíces colonizados) no puede estimarse y sólo se valora cuando se cuantifica la colonización-de las plantas crecidas en el bioensayo. Para verificar la infectividad se realizaron 3 diluciones de suelo de cada situación (v/v $1: 0 ; 1: 4$ y $1: 40)$ en perlita-vermiculita estéril (v/v $1: 1)$ y 12 repeticiones (Díaz \& Honrubia, 1993). Se utilizó como planta hospedadora Medicago sativa L. (alfalfa), dado que es un buen hospedante para hongos formadores de micorrizas y produce una respuesta positiva a la inoculación micorrícica (Cabello, 1997). Se colocaron 2 plántulas por maceta (de un total de180 macetas), las cuales fueron alojadas en un invernáculo a $24^{\circ} \pm 2^{\circ} \mathrm{C}$, con un fotoperíodo de 16 horas de luz provistas por lámparas incandescentes blanco-frías. Para observar el estado de la micorrización, se realizaron 3 cortes a los 15,30 y 60 días de iniciado el ensayo, en los que se tomaron al azar 4 macetas; se tiñó el sistema radical completo de ambas plantas (Phillips \& Hayman, 1970) para evaluar los porcentajes de colonización mediante el método de Giovannetti \& Mosse (1980).

Aislamiento, identificación y análisis de la biodiversidad. Se realizaron ocho muestreos de campo (febrero, abril, junio, agosto, octubre, diciembre - 2003 - febrero, mayo - 2004 -). Tres muestras de cada situación fueron analizadas mediante las técnicas de tamizado y decantado antes descriptas para estimar el número de esporas y las especies fúngicas presentes. Las esporas fueron conservadas en preparados permanentes en alcohol polivinílico (Omar et al., 1979), con y sin reactivo de Melzer (Morton, 1988).

Se adoptó el criterio taxonómico de Schüßler et al., (2001); para la identificación de las especies se siguió a Perez \& Schenck (1988) y a la colección Internacional de hongos formadores de micorrizas vesículo arbusculares (INVAM, http://invam.caf.wvu.edu).

Se calculó la densidad total de esporas considerada como el número total de esporas en $100 \mathrm{gr}$ de suelo seco y la densidad específica de esporas como el número de esporas de cada especie fúngica en 100 gr de suelo seco (Lugo \& Cabello, 2002).

La densidad específica de esporas fue utilizada para calcular el índice de biodiversidad $(H)$ de Shannon Weaver (Magurran, 1988):

$$
H=\prod_{i=1}^{S} \underline{p}_{i}\left(\log _{2} p_{i}\right)
$$

siendo $p_{i}$ la probabilidad de encontrar cada especie $i$ en una muestra

Análisis estadísticos: La densidad de esporas se analizó mediante un ANOVA y LSD $(\mathrm{p}=0.05)$.

\section{RESULTADOS}

1.- Bioensayo de infectividad. El número inicial de esporas de los suelos utilizados en el bioensayo (muestra correspondiente a febrero 2004) fue mayor en las áreas desmontadas respecto de las zonas de bosque nativo. El 
Tabla 1. Contribución de las especies de hongos formadores de micorrizas -arbusculares al índice de biodiversidad $(\mathrm{H})$ en las distintas situaciones: bosque puro de tala $(\mathrm{T})$, bosque puro de coronillo (C), bosque con codominancia de tala y coronillo (TC), desmonte antiguo (DA), desmonte reciente (DR).

\begin{tabular}{|c|c|c|c|c|c|}
\hline Especies & $\mathbf{T}$ & $\mathrm{c}$ & TC & DA & DR \\
\hline Acaniospori delicatir & 0,33 & 0,44 & 0,33 & 0,27 & 0,16 \\
\hline A. lacunosa & 0 & 0 & 0,02 & 0,04 & 0,04 \\
\hline A. igevis & 0 & 0 & 0 & 0,03 & 0,02 \\
\hline A. mellen & 0,21 & 0,50 & 0,44 & 0,38 & 0,37 \\
\hline A. scrobiculis 空 & 0 & 0 & 0 & 0,11 & 0,03 \\
\hline A. spinosi & 0 & 0 & 0,02 & 0,01 & 0 \\
\hline Aca wiospora sp. 1 & 0,44 & 0,05 & 0,09 & 0,02 & 0,01 \\
\hline Aca ulospora sp. 2 & 0 & 0 & 0 & 0,01 & 0 \\
\hline Acs worpors sp. 3 & 0 & 0 & 0,07 & 0 & 0 \\
\hline Entrophospors inflequens & 0,01 & 0,14 & 0,27 & 0,26 & 0,21 \\
\hline Gloms ag greg atum & 0 & 0 & 0 & 0 & 0 \\
\hline G. corbdonium & 0 & 0,07 & 0 & 0 & 0 \\
\hline G. dinum & 0,44 & 0,32 & 0,41 & 0,52 & 0,48 \\
\hline G. constictum & 0,02 & 0,49 & 0,50 & 0,35 & 0,30 \\
\hline G. coronstum & 0,13 & 0,29 & 0,20 & 0,38 & 0,32 \\
\hline G. exuricatum & $i$ & 0,11 & 0,06 & 0,03 & 0,04 \\
\hline G. flstulosum & 0 & 0 & 0 & 0 & 0,01 \\
\hline G. microsgaregatum & 0,05 & 0,03 & 0,06 & 0,04 & 0 \\
\hline G. mosse & 0,02 & 0,11 & 0,09 & 0,07 & 0,14 \\
\hline Gromusp. 1 & 0 & 0 & 0 & 0 & 0 \\
\hline Glomusp. 2 & 0,02 & 0 & 0 & 0,02 & 0,06 \\
\hline Gomas sp. 3 & 0 & 0 & 0,10 & 0 & 0 \\
\hline Glomas sp. 4 & 0 & 0 & 0 & 0 & 0 \\
\hline Sclerocystis sp. & 0,11 & 0,26 & 0,32 & 0,11 & 0,11 \\
\hline ScuteIJospora gilmorei & 0 & 0 & 0,02 & 0,02 & 0,02 \\
\hline ScuteIJospora sp I & 0 & 0 & 0 & 0 & 0,01 \\
\hline ScuteI̋ospora sp 3 & 0 & 0 & 0 & 0 & 0,01 \\
\hline Biodiversidad (H) & 1,85 & 2,84 & 3,018 & 2,73 & 2,42 \\
\hline
\end{tabular}

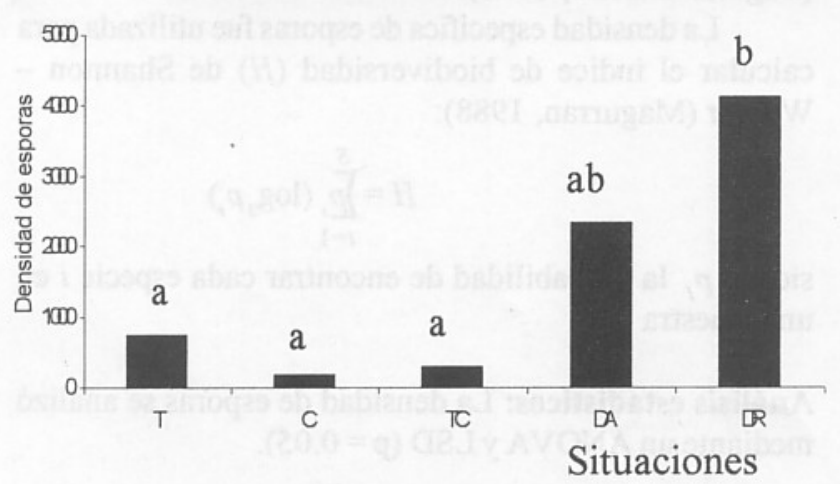

Figura 1. Número de esporas en 100 gr de suelo seco en las distintas situaciones: bosque puro de tala $(\mathrm{T})$, bosque puro de coronillo $(C)$, bosque con codominancia de tala y coronillo (TC), desmonte antiguo (DA), desmonte reciente (DR). Diferentes letras en las columnas muestran diferencias significativas (LSD $\leq \mathbf{0 . 0 5}$ ). Los datos son promedios de 3 repeticiones (bloques). número mínimo fue de 202 esporas en el bosque de coronillo y 3.158 el máximo para el desmonte reciente, siendo este último significativamente diferente de las demás situaciones (Fig. 1).

La colonización presentó valores bajos a los 15 días en las diluciones 1:40 y 1:4 y un valor mayor en el suelo no diluido (1:0) en todos los tratamientos. A los 30 días se observó un aumento pronunciado en todas las diluciones y tratamientos, manteniéndose estos valores estables hasta el último corte a los 60 días (Fig. 2).

2.- Aislamiento, identificación y análisis de la biodiversidad. Se determinaron 26 taxa fúngicos en los distintos tratamientos, pertenecientes a las familias: Glomeraceae, Acaulosporaceae y Gigasporaceae. Glomus clarum, Acaulospora delicata, Acaulospora $\mathrm{sp}$. $1, A$. mellea, G. constrictum y $G$. coronatum, fueron las especies que alcanzan mayores valores de abundancia 


\section{Bosque de tala}

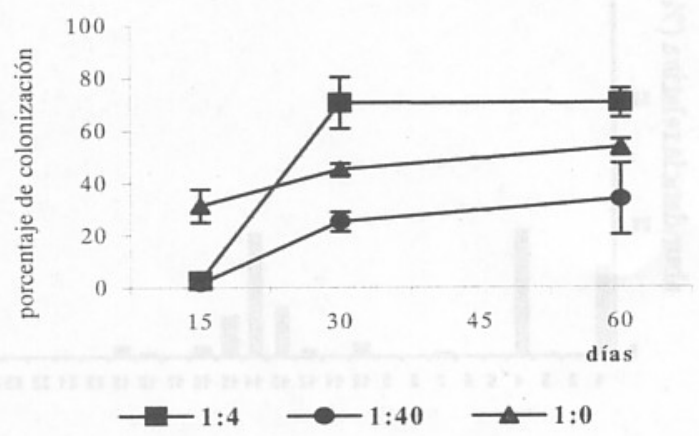

Bosque de tala y coronillo

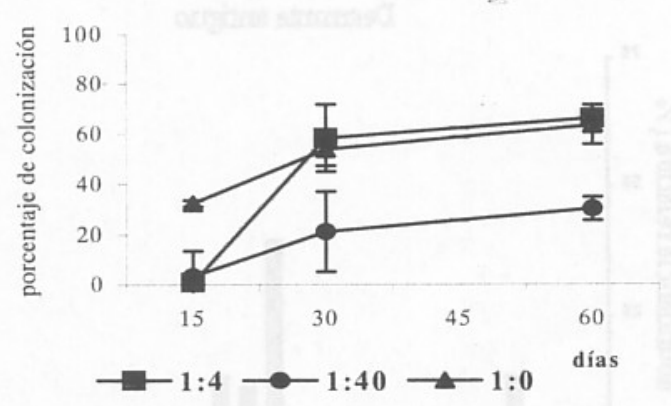

Bosque de coronillo

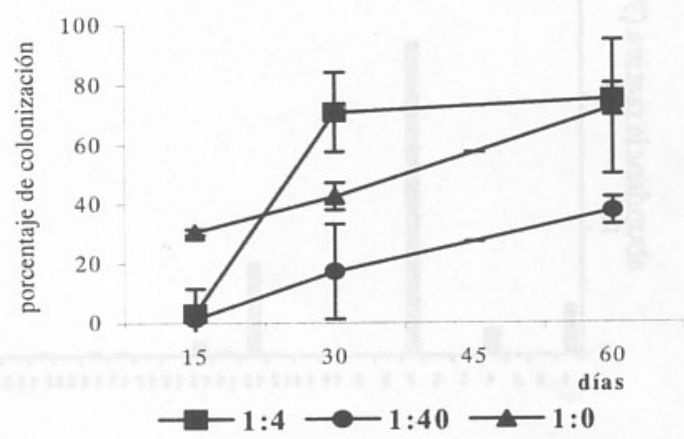

Desmonte antiguo

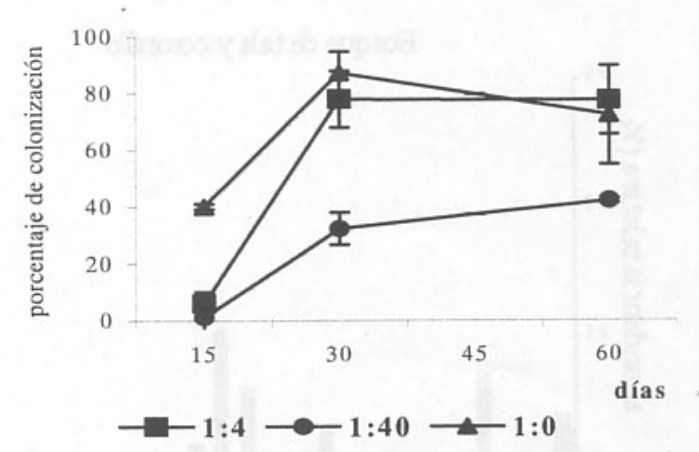

\section{Desmonte reciente}

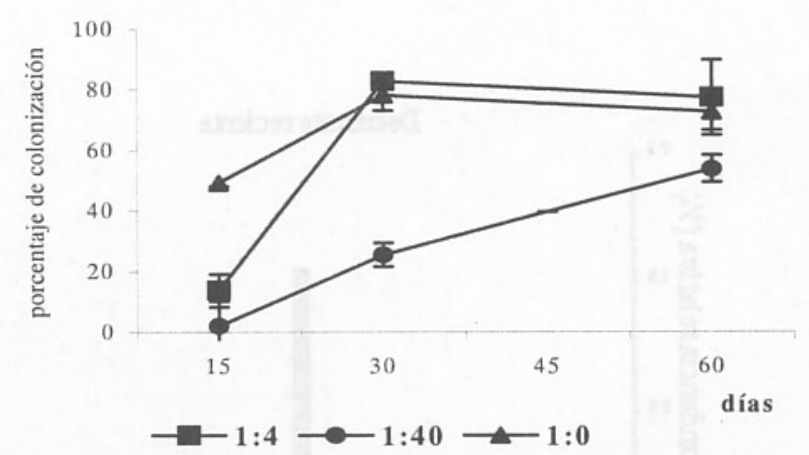

Figura 2. Porcentaje de colonización a los 15, 30 y 60 días en las distintas situaciones (misma simbología de Fig.1). Los datos son promedio de 4 repeticiones, las líneas sobre los puntos indican la desviación estandar.

relativa. La mayoría de las especies presentaron mayor abundancia en las áreas desmontadas con excepción de Acaulospora sp. 1, que tuvo un alto número de esporas en el bosque de tala (Fig. 3).

Las 2 familias con mayor representación específica en todas las áreas y estaciones del año fueron Acaulosporaceae y Glomeraceae, y una muy pequeña contribución de la familia Gigasporaceae presentes únicamente en verano e invierno en la zona recientemente desmontada (Fig. 4). En los sitios desmontados el mayor porcentaje de esporas correspondió a especies pertenecientes a la familia Glomeraceae; en los sitios con cubierta forestal presentó mayor proporción de especies la familia Acaulosporaceae.

La Tabla 1 muestra la contribución de las distintas especies a la biodiversidad total de las diferentes áreas. Glomus clarum, Acaulospora mellea, A. delicata y $G$. coronatum contribuyeron en las 5 situaciones; Acaulospora sp. 1 tuvo un alta contribución en el bosque puro de tala; Sclerocystis sp. estuvo presente en todas las 

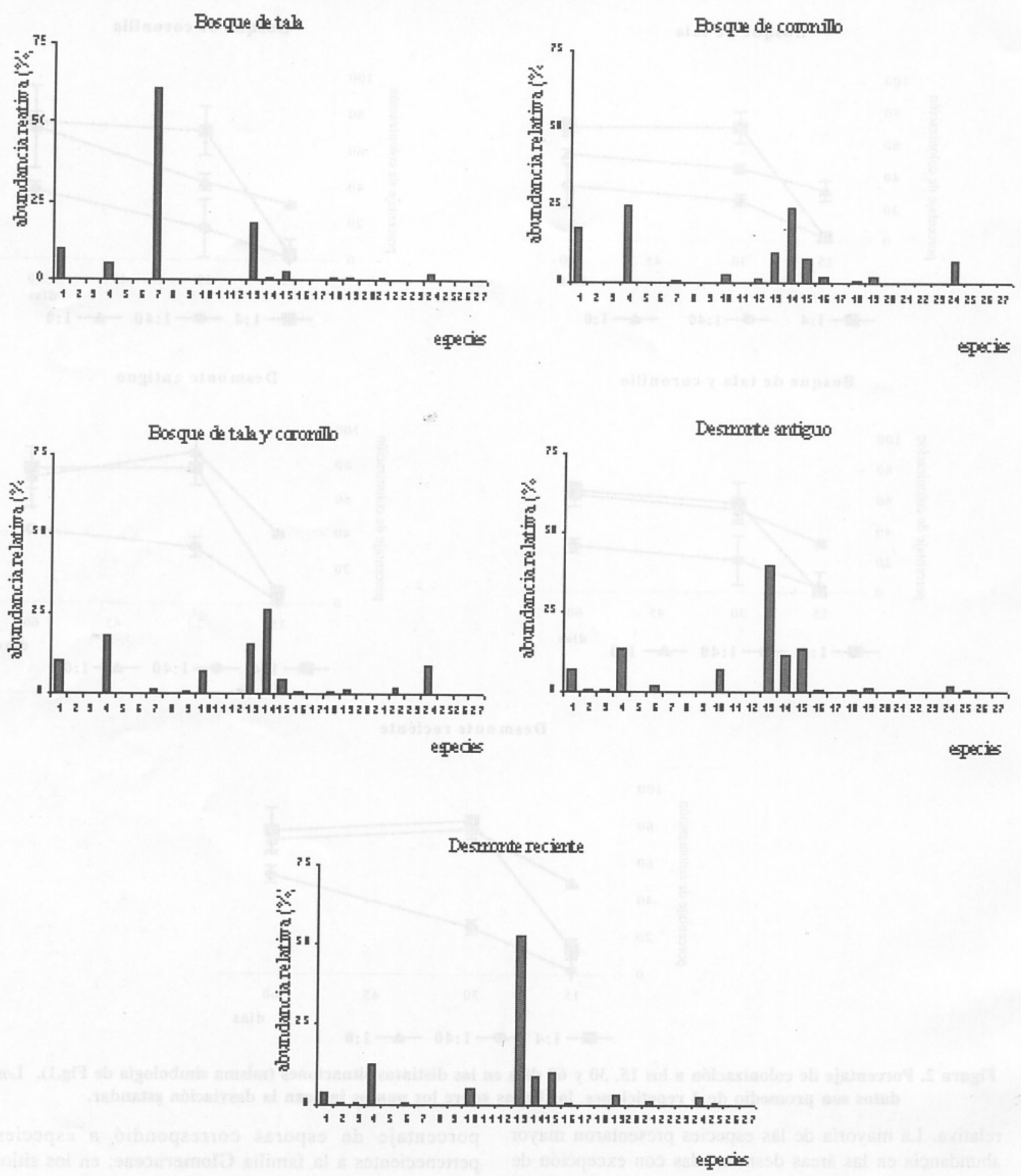

Figura 3. Porcentaje de abundancia relativa de las distintas especies: Acaulospora delicata (1), A. lacunosa (2), A. laevis (3), A. mellea (4), A. scrobiculata (5), A. spinosa (6), Acaulospora sp. 1 (7), Acaulospora sp. 2 (8), Acaulospora sp. 3 (9), Entrophospora infrequens (10), Glomus aggregatum (11), G. caledonium (12), G. clarum (13), G. constrictum (14), G. coronatum (15), G. etunicatum (16), G. fistulosum (17), G. microaggregatum (18), G. mosseae (19), Glomus sp. 1 (20), Glomus sp. 2 (21), Glomus sp. 3 (22), Glomus sp. 4 (23), Sclerocystis sp. (24), Scutellospora gilmorei (25), Scutellospora sp. 1 (26), Scutellospora sp. 2 (27); en cada situación. 
Verano

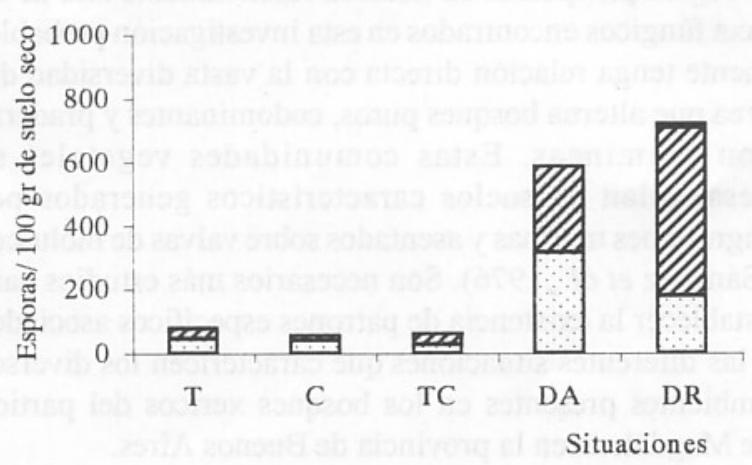

Invierno

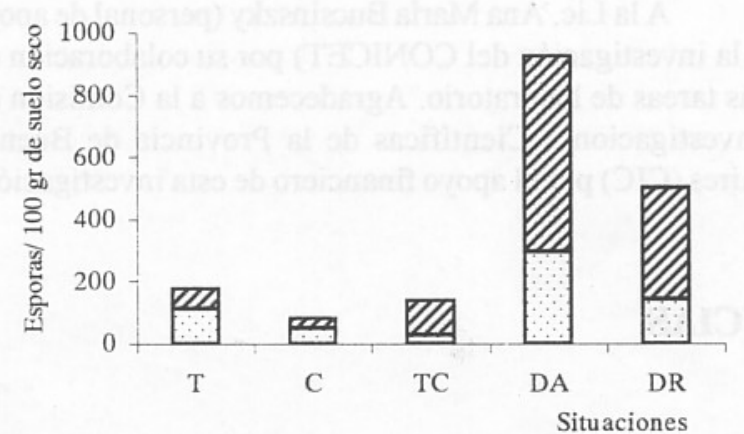

Glomeraceae
Situaciones

Gigasporaceae
Otoño

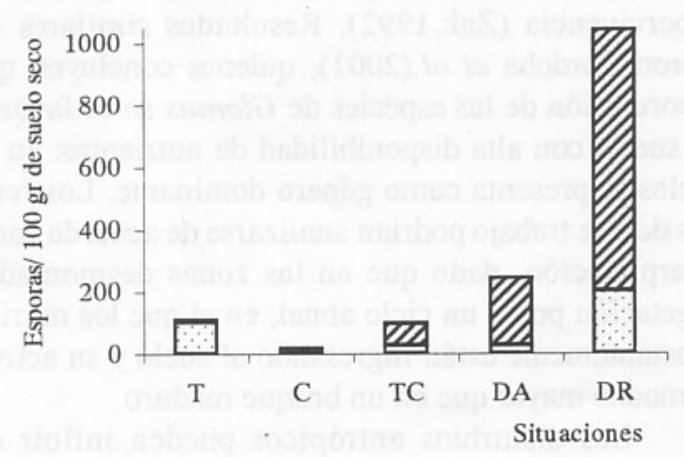

Primavera

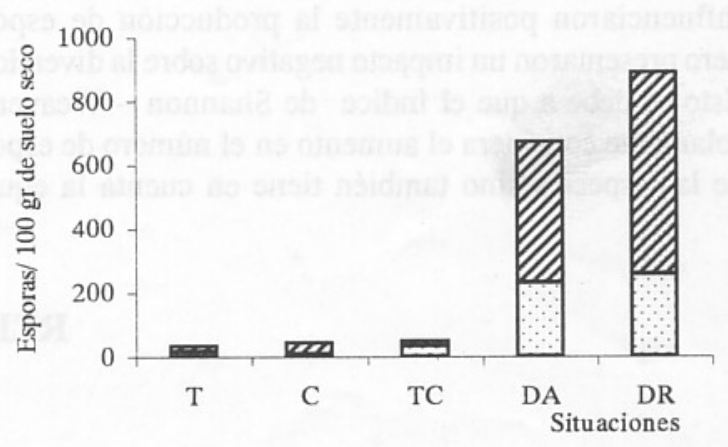

Acaulosporaceae

Figura 4. Abundancia estacional de esporas en $100 \mathrm{~g}$ de suelo seco y su contribución a la familias Acaulosporaceae, Glomeraceae y Gigasporaceae en las distintas situaciones (misma simbología de Fig.1). Los datos son promedio de los muestreos de agosto, octubre (2003), febrero y mayo (2004).

situaciones pero presentó mayor contribución en los bosques de tala y coronillo. Otras especies sólo estuvieron representados en algunas situaciones, tal es el caso de Scutellospora gilmorei, presente en bosques codominantes y desmontes, $\boldsymbol{A}$. scrobiculata únicamente en los desmontes y Acaulospora sp. 3 y Glomus sp. 4 sólo en el bosque con codominancia de tala y coronillo.

El valor del índice de biodiversidad para cada situación fue mayor en el bosque con codominancia de tala y coronillo y en el bosque puro de coronillo, los desmontes presentaron valores más bajo y el menor valor se registró en el bosque puro de tala (Tabla 1).

\section{DISCUSION}

La infectividad no mostró diferencias en los 5 suelos probados, contrastando con la variación presente en el número de esporas. Esta diferencia indica que no existe relación entre el número de esporas y la infectividad. Iguales resultados fueron obtenidos por Díaz \& Honiubia (1993). Existen diversos factores que influyen . en las tasas primarias de infección, uno de ellos es la dormancia de las esporas, la capacidad de las especies de poseer un desarrollo saprotrófico en el suelo, la cantidad y velocidad en la formación de apresorios.

Bajo ciertas condiciones, la esporulación puede llegar a cero, como es el caso de los bosques siempreverdes tropicales en los cuales existe una alta tasa de infección (Sieverding, 1991). En nuestro caso, al igual que en los bosques tropicales, la disponibilidad constante de fuentes carbonadas en las áreas arboladas a través de las raíces de los árboles haría que la principal fuente de propágulos corresponda al micelio externo e interno de las raíces colonizadas con baja producción de esporas, mientras que en las zonas desmontadas la disponibilidad de fotoasimilados depende de plantas mayormente herbáceas y anuales. 
La presencia de más representantes de la familia Glomeraceae en las áreas desmontadas, indicaría una adaptación de estas especies, mediante una alta y muy rápida producción de esporas como estrategia básica de supervivencia (Zak, 1992). Resultados similares obtuvieron Cordoba et al.(2001), quienes concluyen que la esporulación de las especies de Glomus se ve favorecida en suelos con alta disponibilidad de nutrientes; en estos suelos se presenta como género dominante. Los resultados de este trabajo podrían analizarse de acuerdo con esta interpretación, dado que en las zonas desmontadas la vegetación posee un ciclo anual, en el que los nutrientes constantemente están ingresando al suelo y su actividad es mucho mayor que en un bosque maduro.

Los disturbios antrópicos pueden influir en la abundancia y la distribución de las esporas de hongos formadores de micorrizas arbusculares (Jasper et al., 1991). En nuestro estudio observamos que los desmontes influenciaron positivamente la producción de esporas pero presentaron un impacto negativo sobre la diversidad. Esto se debe a que el índice de Shannon - Weaver no solamente considera el aumento en el número de esporas de las especies sino también tiene en cuenta la equita- tividad de su distribución en la muestra (Frontier \& PichoViale, 1995). Menéndez et al.(2001), encontraron 17 especies de hongos micorrícicos en suelos agrícolas de la región pampeana. El número relativamente alto de 26 taxa fúngicos encontrados en esta investigación probablemente tenga relación directa con la vasta diversidad del área que alterna bosques puros, codominantes y praderas con gramíneas. Estas comunidades vegetales se desarrollan en suelos característicos generados por ingresiones marinas y asentados sobre valvas de moluscos (Sánchez et al., 1976). Son necesarios más estudios para establecer la existencia de patrones específicos asociados a las diferentes situaciones que caractericen los diversos ambientes presentes en los bosques xerícos del partido de Magdalena en la provincia de Buenos Aires.

\section{AGRADECIMIENTOS}

A la Lic. Ana María Bucsinszky (personal de apoyo a la investigación del CONICET) por su colaboración en las tareas de laboratorio. Agradecemos a la Comisión de Investigaciones Científicas de la Provincia de Buenos Aires (CIC) por el apoyo financiero de esta investigación.

\section{REFERENCIAS}

Allen, M.F. (1991). The ecology of mycorrhizae. En: De Barnes, R.S.K., Birks, H.J.B., Connor, E.F., Harper J.L. \& Paine, R. (eds.) Cambridge, New York, Port Chester, Melbourne, Sydney: Cambridge University.

Augé, R.M. (2001). Water relations, drought and vesicular-arbuscular mycorrhizal symbiosis. Mycorrhiza. 11:3-42

Bouhot, D. (1980). Le potentiel infectieux des sols: un concept, ur: modèle pour sa mesure, quelques applications. Thèse de doctoral. Université de Nancy I, Nancy, France.

Cabello, M.N. (1997). Hydrocarbon pollution: its effect on native arbuscular mycorrhizal fungi (AMF). FEMS Microbiol. Ecol. 22: 233-236

Cabrera, A.L. (1968). Flora de la Provincia de Buenos Aires. Colección Cientifica del INTA, Buenos Aires. pp. 33-101

Cordoba, A.S.; De Mendonça, M.M.; Stürmer, S.L. ; Rygiewicz, P.T. (2001). Diversity of arbuscular mycorrhizal fungi along a sand dune stabilization gradient: A case study at Praia de Joaquina Ilha de Santa Catarina, South Brazil. Mycoscience 42: 379-387

Diaz, G. \& Honrubia, M. (1993). Infectivity of mine soils from Southest Spain. II Mycorrhizal population levels in spoilt sites.Mycorrhiza 4:85-88

Fidalgo, F.; De Francesco, F.O. \& Pascual, R. (1975). Geología superficial de la llanura bonaerense, Argentina. Relatorio VI Congreso Geol. Arg., Bahía Blanca.

Frontier, S. \& Pichod -Viale, D. (1995). Écosystèmes. Structure, fonc- tionnement, évolution. Masson, Paris. pp. 290-311

Gerdemann, J.W. \& Nicoloson, T.H. (1963). Spores of mycorrhizal Endogone species extracted from soil by wet sieving and decanting. Trans. Br. mycol. Soc. 84: 679-684

Giovannetti, M. \& Mosse, B. (1980). An evaluation of techniques for measuring vesicular-arbuscular mycorrhizal infection in roots. New Phytol. $84: 489-499$

Goya, J. F.; Placci, L. G.; Arturi, M. F. \& Brown, A. D. (1992). Distribución y características estructurales de los talares de la Reserva de Biósfera "Parque Costero del Sur". Rev. de la Fac. de Agron. La Plata. 68: 53-64

Grime, J.P.; Mackey, J.M.L.; Hillier, S.H. \& Visser S.A. (1987). Floristic diversity in a model system using experimental microcosm. Nature, London 328: $420-422$

Hetrick, B.A. (1984). Ecology of VA mycorrhizal fungi. En: Powell, C.L. \& Bagyaraj D.J. eds. VA mycorrhiza. CRC Press, Boca Raton, Florida. pp 3355

Jasper, D.A.; Abbott, L.K. \& Robson, A.D. (1991). The effect of soil disturbance on vesicular-arbuscular mycorrhizal fungi in soil from different vegetation types. New Phytol. 118: 471-476

Johnson, N.C. \& Pfleger, F.L. (1992). Vesicular-arbuscular mycorrhizae and cultural stresses. En: Bethlenfalvay, G.J. \& Linderman R.G. (eds.) Mycorrhiza in sustainable agriculture. Am. Soc. Agron. Spec. Publ. 54, Madison, Wisconsin. pp 71-99 
Lugo, M.A \& Cabello, M.N. (2002). Native arbuscular mycorrhizal fungi (AMF) from mountain grassland (Córdoba, Argentina) I. Seasonal variation of fungal spore diversity. Mycologia 94: 579-586

Magurran, A.E. (1988). Ecological diversity and its measurement. Croom Helm, London.

Menéndez, A. B.; Scervino, J. M. \& Godeas, A. M. (2001). Arbuscular mycorrhizal populations associated with natural and cultivated vegetation on a site of Buenos Aires province, Argentina. Biol. Fertil. Soils 33:373381

Miller, S.L. \& Allen, E.B. (1992). Mycorrhizae, nutrient translocation, and interactions between plants. En: Allen, M.F Mycorrhizal Functioning (ed.). Chapman \& Hall: New York, USA. pp 301-332

Molina, R. ; Massicotte, H. \& Trappe, J.M. (1992). Specificity phenomena in mycorrhizal symbioses: community-ecological consequences and practical implications. En: Allen, M.F. (ed.) Mycorrhizal Functioning. Chapman \& Hall: New York, USA.pp 357- 423

Morton, J. B. (1988). Taxonomic of VA mycorrhizal fungi: classification, nomenclature and identification. Mycotaxon 32: 267-324

Omar, M.B.; Bolland, L. \& Heather, W.A. (1979). PBA (polivinil alcohol). A permanent mounting medium for fungi. Bull. Brit. Mycol. Soc. 13: 31-32
Phillips, J.M. \& Hayman, D.S. (1970). Improved procedures for clearing roots and staining parasitic and vesicular-arbuscular mycorrhizal fungi for rapid assessment of infection. Trans. Br. Mycol. Soc. 55: 158-161

Sánchez, R.O. ; Ferrer, J.A. ; Duymovich, O.A. \& Hurtado, M.A. (1976). Estudio pedológico integral de los Partidos de Magdalena y Brandsen (Provincia de Buenos Aires). Anales del LEMIT, Serie II, 310. Ministerio de Obras Públicas de la provincia de Buenos Aires.

SchüBler, A.; Schwarzott, D. \& Walker, C. (2001). A new fungal phylum, the Glomeromycota: phylogeny and evolution. Mycol. Res. 105: 14131421

Sieverding, E. (1991). Vesicular-Arbuscular Mycorrhiza Management in Tropical Agrosystems. Deutsche Gesellschaft für Technische Zusammenarbeit: Eschborn, Germany.

Walker, C.; Mize, W. \& McNabb, H.S. (1982). Populations of endogonaceous fungi at two populations in Central Iowa. Can J. Bot. 60: 2518-2529

Zak, J.C. (1992). Response of soil Fungal Communities to disturbances. En: Carroll G.C \& Wicklow D.T. (eds.) The fungal community. Its organization and role in the ecosystem. Marcel Dekker, USA. pp. 403-425 